\title{
H I Lyman-alpha equivalent widths of stellar populations
}

\author{
María A. Peña-Guerrero ${ }^{1}$ \\ pena@stsci.edu \\ and \\ Claus Leitherer ${ }^{1}$ \\ leitherer@stsci.edu
}

\begin{abstract}
We have compiled a library of stellar Ly $\alpha$ equivalent widths in $\mathrm{O}$ and $\mathrm{B}$ stars using the model atmosphere codes CMFGEN and TLUSTY, respectively. The equivalent widths range from about 0 to $30 \AA$ in absorption for early-O to mid$\mathrm{B}$ stars. The purpose of this library is the prediction of the underlying stellar Ly $\alpha$ absorption in stellar populations of star-forming galaxies with nebular Ly $\alpha$ emission. We implemented the grid of individual equivalent widths into the Starburst99 population synthesis code to generate synthetic Ly $\alpha$ equivalent widths for representative star-formation histories. A starburst observed after $10 \mathrm{Myr}$ will produce a stellar Ly $\alpha$ line with an equivalent width of $\sim-10 \pm 4 \AA$ in absorption for a Salpeter initial mass function. The lower value (deeper absorption) results for an instantaneous burst, and the higher value (shallower line) for continuous star formation. Depending on the escape fraction of nebular Ly $\alpha$ photons, the effect of stellar Ly $\alpha$ on the total profile ranges from negligible to dominant. If the nebular escape fraction is $10 \%$, the stellar absorption and nebular emission equivalent widths become comparable for continuous star formation at ages of 10 to 20 Myr.
\end{abstract}

Subject headings: star-forming galaxies

\section{Introduction}

Galaxies with active star formation host large populations of massive, hot, young $\mathrm{O}$ and B stars which ionize the surrounding interstellar medium (ISM). The resulting nebular

${ }^{1} 3700$ San Martin Drive, Baltimore, MD, 21218, USA 
recombination spectrum includes numerous strong emission lines which are widely used as star-formation tracers (Kennicutt \& Evans 2012). Among these lines, Lyman-alpha (Ly $\alpha$ ) deserves special attention. Ly $\alpha$ photons are expected to be produced when the opacity approaches infinity for those photons associated with the base level (Case B). Hence, if Case B applies, strong Ly $\alpha$ emission is expected, and this line can be detected even at the highest redshifts (i.e. Partridge \& Peebles 1967; Malhotra \& Rhoads 2002; Stern et al. 2005; Bromm \& Yoshida 2011; Sandberg et al. 2013, and references therein).

At high redshift Ly $\alpha$ is often the only detectable emission line in the optical and nearinfrared, and is therefore widely used as an indicator of star formation (i.e. Malhotra \& Rhoads 2002; Scarlata et al. 2009; Dijkstra \& Wvithe 2012). If the continuum light is detected, the Ly $\alpha$ equivalent width $(\mathrm{EW})$ can give valuable clues on population ages and the stellar initial mass function (IMF) (Leitherer et al. 2010). If Case B applies, the theoretically predicted values of EW are expected to vary from 50 to $\sim 240 \AA$ in star-forming galaxies with ages of less than 100 Myr (i.e. Charlot \& Fall 1993; Laursen. Duval \& Östlin 2013, and references therein). However, Ly $\alpha$ turns out to be a complex star-formation tracer. In most cases the observed line strength is less than expected from Case B recombination because the effects of radiation transfer have not been taken into account (Hansen \& Oh 2006). The assumption of Ly $\alpha$ being a pure recombination line in a gaseous medium is too simple. Meier \& Terlevich (1981), Hartmann et al. (1988), Neufeld (1990), and Charlot \& Fall (1993) considered the effects of dust on the Ly $\alpha$ radiative transfer. Resonant scattering by atomic hydrogen significantly increases the path length for Ly $\alpha$ photons and consequently the likelihood of Ly $\alpha$ destruction by dust, leading to much lower line strengths. On cosmological scales, models incorporating a redshift-dependent intergalactic dust obscuration (Haiman \& Spaans 1999; Malhotra \& Rhoads 2004) predict Ly $\alpha$ space densities that are broadly consistent with "semi-analytical" models connecting Ly $\alpha$ emitting galaxies and dark matter halos.

Although there is consensus that Ly $\alpha$ is regulated by dust, no clear correlation has been found between dust content and Ly $\alpha$ EW at low redshift (z) Giavalisco, Koratkar \& Calzetti 1996; Hansen \& Oh 2006). However, at $z \sim 3$ the picture seems to be rather different. Shapley et al. (2003) found a significant correlation between UV continuum extinction and Ly $\alpha$ EW. They suggest that this difference between the low and high redshift could be due to either differences in the geometry of dust in the neutral gas, or small sample statistics biases at low redshift. Furthermore, Laursen. Duval \& Östlin (2013) found that different ISM morphologies are unlikely to account for different Ly $\alpha$ escape probabilities. Instead, they propose an increase of Ly $\alpha$ radiation due to cold accretion and/or anisotropic escape. Most importantly, the kinematic properties of the ISM may very well be the dominant escape or trapping mechanism for Lya (Kunth et al. 2003; Fujita et al. 2003; Mas-Hesse et al. 2003; Dijkstra \& Wvithe 2010). 
In addition to physical processes related to the ISM, the observed Ly $\alpha$ emission can be modified by corrections for any underlying stellar Ly $\alpha$. Depending on the stellar population properties, these corrections can be quite substantial and even cancel out the nebular emission. Valls-Gabaud (1993) suggested stellar Ly $\alpha$ absorption from an aged population as the reason for the dearth of Ly $\alpha$ emitters among local starburst galaxies.

The stellar Ly $\alpha$ cannot be fully constrained observationally since even the closest $\mathrm{O}$ stars in our Galaxy have ISM H I column densities high enough to produce strong interstellar Ly $\alpha$, which masks their total intrinsic stellar line. In this work we retrieved publicly available theoretical spectra of O and B stars generated with CMFGEN (Hillier \& Miller 1998; Hillier 2011) and TLusty (Hubeny \& Lanz 1995; Lanz \& Hubeny 2003, 2007), respectively, and determined 276 individual Ly $\alpha$ EW's. The EW's are used as a library for implementation in the Starburst99 synthesis code (Leitherer et al. 1999; Leitherer \& Chen 2009). The goal of our work is to predict the behavior of the stellar $\operatorname{Ly} \alpha$ as a function of population properties. While understanding the behavior of stellar Ly $\alpha$ is crucial in its own right, we are mainly motivated by the need to evaluate the effect of the stellar contribution to the total Ly $\alpha$ emission in star-forming galaxies. This work is organized as follows. In Section 2 we present a description of the models used for the new theoretical library, and we describe how we determined EW's. In Section 3 we compare our determinations with observations of individual $\mathrm{O}$ and $\mathrm{B}$ stars, and in Section 4 we describe the implementation of the new library in the Starburst99 code and the resulting EW's of typical populations. In Section 5 we present our conclusions.

\section{Methodology}

Our goal is to predict the stellar Ly $\alpha$ line strength of a population of young stars whose ionizing photons give rise to the observed nebular Ly $\alpha$ emission. The relevant stars are of spectral types $\mathrm{O}$ and $\mathrm{B}$. Later types contribute negligible light at $1216 \AA$ in a population with OB stars present. Alternatively, a single, evolved population at an age older than $\sim 100$ Myr (when A stars start to dominate) would not be observed as a Ly $\alpha$ emitter because of the dearth of ionizing photons.

Strong stellar winds are a defining characteristic of OB stars. The spectral signatures of these winds are blueshifted absorption, broad emission, or P Cygni-type profiles (Puls, Vink, \& Najarro 2008). Ly $\alpha$ in particular, is susceptible to wind effects due to its formation depth far above the photosphere. This was already recognized during the early efforts of non-Local Thermal Equilibrium (non-LTE) modeling by Klein \& Castor (1978) who emphasized that the strength of Ly $\alpha$ in luminous $\mathrm{O}$ stars is never significant due to 
canceling effects of the emission and absorption components.

In this work we utilize stellar spectra of O stars computed with the CMFGEN atmosphere code, which was designed to solve the radiative transfer and statistical equilibrium equations in hot stars in spherical geometry (Hillier \& Lanz 2001). The models account for non-LTE and are fully blanketed. The CMFGEN code was originally written for modeling the strong winds of Wolf-Rayet stars (Hillier 1987), but has since been generalized to be applicable to O stars as well. Pre-calculated grids of O-star models are readily available in various databases so that there is no need to generate additional models. We surveyed the available spectra of O stars from the Pollux database (Palacios et al. 2010), and retrieved 46 CMFGEN O star spectra of solar metallicity, microturbulent velocity of 5 and $10 \mathrm{~km} / \mathrm{s}$, effective temperatures $\left(T_{\text {eff }}\right)$ ranging from 27,500 to $48,530 \mathrm{~K}$, and surface gravity $(\log g$ ) ranging from 3.0 to 4.25. The mass-loss rate used in this O-grid ranged from $4.85-8.34 \times 10^{-6} \mathrm{M}_{\odot} / \mathrm{yr}$, and has been adopted to match the observed rates. A $\beta$-velocity law (Lamers \& Cassinelli 1999) was adopted, with $\beta=0.8$. The O-grid available on the CMFGEN webpage 1 is a subset of that contained in the Pollux database and therefore provides no additional new data (the O-grid in the CMFGEN website contains only 23 models of O stars).

Hot-star winds scale with luminosity and (to a smaller degree) with $T_{\text {eff }}$ (Vink 2007). As a result, wind effects become less pronounced in $\mathrm{B}$ stars when compared to $\mathrm{O}$ stars. B star spectra show less evidence for winds, except for the most luminous supergiants, which are rare by number in a typical stellar population. Given this situation, we opted for Bstar models based on the TLUSTY atmosphere code (Lanz \& Hubeny 2007). Like CMFGEN, TLUSTY is a fully metal-blanketed non-LTE code. In contrast to CMFGEN, TLUSTY adopts a static, plane-parallel geometry, which does not account for stellar-wind effects above the photosphere. TLUSTY is an excellent representation of B main-sequence stars and of evolved B stars with moderate winds. Modeled spectra of B stars were obtained from the TLUSTY website 2 . We used the BSTAR2006 grid of B stars with solar metallicity. We retrieved 212 modeled B star spectra with microturbulent velocities of 2 and $10 \mathrm{~km} / \mathrm{s}, T_{\text {eff }}$ ranging from 15,000 to $30,000 \mathrm{~K}$, and $\log g$ ranging from 1.75 to 4.75 . The specific characteristics of the models are presented in Lanz \& Hubeny.

Since winds are not completely absent in B-type stars, we need to be concerned about the applicability of the static, TLUSTY models. Heap, Lanz, \& Hubeny (2006) provide an extensive discussion of the trades between the CMFGEN and TLUSTY codes. While the preference for spherical, expanding atmospheres in $\mathrm{O}$ stars is obvious, it is preferable to

\footnotetext{
${ }^{1}$ http://kookaburra.phyast.pitt.edu/hillier/web/CMFGEN.htm

${ }^{2}$ http://nova.astro.umd.edu/index.html
} 
use sophisticated photospheric models such as TLUSTY for stars with moderate or weak winds rather than rely on spherical models with a simplified physics of the deep, quasi-static layers. This justifies our choice of CMFGEN for O stars, and TLUSTY for B stars.

Massey et al. (2013) found that the choice of different microturbulent velocities has little effect on heavy element lines and no effect on the hydrogen lines. We measured the difference in the determined EW's from models with different microturbulent velocities. We found that the mode on this difference was $\sim 3 \AA$, and the minimum and maximum differences were 0.1 and $7.2 \AA$, respectively. We used the microturbulence velocity of $10 \mathrm{~km} / \mathrm{s}$ unless this value was not available. In Table 1 we give a summary of the models compiled for this work. There is a total of 258 model spectra considering all microturbulent velocities. The parameters cover the full observed range of $T_{\text {eff }}$ and $\log g$ in the upper Hertzsprung-Russell diagram.

In order to obtain determinations of $\operatorname{Ly} \alpha \mathrm{EW}, 3$ comparable to those obtained from observations, we rebinned the spectra from the extremely high resolution of the models ( $\Delta \lambda$ of $0.060 \AA$ for CMFGEN and $0.005 \AA$ for TLUSTY at Ly $\alpha$ ) to $\Delta \lambda$ of $0.465 \AA$. This was done using a cubic spline function. This value is an average of $\Delta \lambda$ of 0.75 and $0.18 \AA$, the typical resolutions of the two spectrographs on board of HST, the Space Telescope Imaging Spectrograph and Cosmic Origins Spectrograph, used for observing extragalactic objects.

Our determinations of EW's were done with a simple flux over continuum integration code written in Python. For the TLUSTY modeled atmospheres, both the continuum and the lines spectra were retrieved from their webpage. Hence, the EW determination was straight forward since the continuum location is predefined. However, for the Pollux retrieved SEDs, only the lines spectra were available. As expected, the EW determination changed with the height where the continuum was placed. The continuum was set to be a straight line between two points free of lines in a wavelength range from 1100 to $1300 \AA$. To test the validity of this method we used the small grid of $O$ stars from the CMFGEN website that do give both the continuum and the lines files. We retrieved these files and normalized the spectra (divided the lines by the continua files). We then determined the EW's with the Python code integrating through $10 \AA$ centered at Ly $\alpha$. For the purpose of comparison we will call these determinations the benchmark EW's. We then used only the lines files to fit our straight line continuum method and determined the EW's with the same Python code. The resulting EW's have on average an error of $1 \%$ and up to $5 \%$ with respect to the benchmark EW's.

Figures 1 and 2 show representative spectra of $\mathrm{O}$ and $\mathrm{B}$ stars, respectively. In both

\footnotetext{
${ }^{3}$ The sign convention used in this work is positive for emission lines and negative for absorption lines.
} 
figures the most prominent lines are marked for reference, and the red horizontal line indicates the position of the continuum and the EW integration range for each star type. These reference lines are: C III $1175 \AA$, Si III $1206 \AA$, H I Ly $1216 \AA$, N V $1240 \AA$, and C III $1247 \AA$. For O stars the spectra of most models show clear P Cygni profiles, indicating the combination of photospheric absorption and high density stellar winds due to radiation pressure (Leitherer et al. 1995). In the spectra of luminous O stars and even in the earliest B stars, the N V line can be quite prominent (and in some cases blend with Ly $\alpha$ ), though its strength decreases for later B stars (Walborn et al. 1985). In turn, the Si III line does not play a major role in the spectra of $\mathrm{O}$ stars but in B stars this line can easily blend with Ly $\alpha$, since the Ly $\alpha$ width increases considerably for later B type stars (Walborn et al. 1995). Since in this study we aim to determine the stellar contribution of the observed Ly $\alpha$ EW, we want to avoid most of the possible blendings with Ly $\alpha$. We therefore chose different EW integration ranges for $\mathrm{O}$ and $\mathrm{B}$ stars.

For the modeled $\mathrm{O}$ stars, the integration was performed over $10 \AA$ centered on the rest frame wavelength of the Ly $\alpha$ line (strictly $1215.67 \AA$ ). The EW's of these stars were on average in absorption and mostly close to $0 \AA$ due to the strong $\mathrm{P}$ Cygni wind profiles (see Figure 1). Hence, the chosen wavelength range of $10 \AA$ allowed us to account for the full width of Ly $\alpha$, including wings and the P Cygni profiles, if present.

For the modeled B stars, the integration was done over $40 \AA$ centered on the rest frame wavelength of Ly $\alpha$ in order to account for the broader Ly $\alpha$ absorption. For clarity we will call this determination "simple integration" EW. Nonetheless, the Si III resonance line at 1206.5 $\AA$ is an important source of line blending for B stars (Savage \& Panek 1974). In order to correct for the contribution of this line to Ly $\alpha$ we assumed that Ly $\alpha$ was symmetric, and avoided the part of the spectrum where Si III and Ly $\alpha$ overlap. We integrated the EW from the rest wavelength of $\operatorname{Ly} \alpha$ to $\operatorname{Ly} \alpha+20 \AA$ and then multiplied this EW by 2 . We call this determination "half integration" EW.

Savage \& Panek (1974) found that the typical contribution of the Si III line to Ly $\alpha$ is about $4 \AA$. When comparing the Ly $\alpha$ EW determinations through "simple integration" EW to the "half integration" EW, we found that the difference between the first and the second EW determination is on average $3 \AA$ but peaks between $\log g$ of 2.0 and 2.50 to about $7 \AA$ below an effective temperature of $21,000 \mathrm{~K}$. We chose the "half integration" EW to better represent the Ly $\alpha$ EW since the Si III line is a clear systematic feature in the modeled B star spectra (see Figure 2). The resulting EW determinations for B stars are all in absorption and range from 1 to $32.5 \AA$.

For $T_{\text {eff }}$ of 15,000 to $30,000 \mathrm{~K}$ we used the TLUSTY models and for $T_{\text {eff }}>30,000 \mathrm{~K}$ we used the CMFGEN models. We did a linear interpolation to obtain the EW's for those 
values of $T_{\text {eff }}$ and $\log g$ that were not included in the model spectra we retrieved. The final EW's adopted in this work are presented in Table 2. Figure 3 shows the trend of our EW determinations with respect to $T_{\text {eff }}$ for different values of $\log g$. We attribute the slight jump in this figure to the different treatments of the stellar winds used in TLUSTY and CMFGEN codes. Nonetheless, in order to check for consistency between both codes, we studied in the differences between EW measurements in the overlapping region (i.e. $T_{\text {eff }}$ between 27,000 and $30,000 \mathrm{~K}$ ). We found that the mode and average difference of the CMFGEN and the TLusty EWs is about $7 \AA$, with a maximum difference of $9 \AA$ at $T_{\text {eff }}$ of $30,000 \mathrm{~K}$ and $\log g$ of 3.75 .

The behavior shown in Figure 3 is consistent with what is expected from observations. For both $\mathrm{O}$ and $\mathrm{B}$ stars, the intensity and width of the ultraviolet features is closely related to the luminosity type (Walborn et al. 1995). Spectra of O stars show a P Cygni profile in some lines (late-B stars for metal lines) when the absorbing ions column density is larger than about $10^{15}$ ions per $\mathrm{cm}^{-2}$ (Lamers \& Cassinelli 1999). This is precisely the overlapping region in our EW measurements, from 27,000 to 30,000 K. The P Cygni profiles for Ly $\alpha$ become progressively smaller as the $T_{\text {eff }}$ decreases, hence making the EW progressively differ from zero (i.e. a greater absorption as the wind emission becomes smaller). As $T_{\text {eff }}$ keeps decreasing, the Ly $\alpha$ absorption becomes wider.

In order to explore how non-solar chemical compositions affect the Ly $\alpha \mathrm{EW}$, we obtained spectra of TLUSTY of B stars with $T_{\text {eff }}$ of $30,000 \mathrm{~K}$ and $\log g$ of 4.25 , and with 4 different metallicities besides solar. These stars are also described in Lanz \& Hubeny (2007). We determined the EW's with the "half integration" method. The determined Ly $\alpha$ EW's for metallicities (in units of $\mathrm{Z} / \mathrm{Z}_{\odot}$ ) of $2,1,1 / 2,1 / 5$, and $1 / 10$ were: $-15.38,-12.87,-10.43$, 9.85, and $-6.30 \AA$, respectively. The overall shape of the spectrum changes very little with metallicity, i.e. the lines around $\operatorname{Ly} \alpha$ become less prominent, making it easier to detect Ly $\alpha$ even considering that the EW is also getting smaller. This behavior is as expected since: (i) the EW is defined as the wavelength range over which the continuum around the line must be integrated to produce the same intensity as the observed line (Lallement et al. 2011), and (ii) the effect of metallicty on other hydrogen lines such as the Balmer lines is also rather weak if the $T_{\text {eff }}$ is higher than 7,000 K (González-Delgado \& Leitherer 1999).

\section{Comparison with Observations of $\mathrm{O}$ and $\mathrm{B}$ stars}

In order to gain confidence into our set of Ly $\alpha$ EW's, we surveyed the literature for available observations of stellar Ly $\alpha$. There is a rich body of data dating back to the early observations by OAO-2 (Savage \& Jenkins 1972), OAO-3 (Bohlin et al. 1978), and IUE 
(Diplas \& Savage 1994a,, b). We compared our theoretical Ly $\alpha$ EW determinations with those from observations of O and B stars presented in Savage \& Code (1970) and Savage \& Panek (1974). The observations presented in Savage \& Code and Savage \& Panek were taken with OAO-2, whose spectrograph had a resolution of $15 \AA$. The relatively low resolution implies that there could be contamination with Si III on the short-wavelength side of Ly $\alpha$ and possibly with $\mathrm{N}$ V on the long-wavelength side, depending on the extent of the wings of Ly $\alpha$.

We adopted the EW's as published by Savage \& Code (1970) and Savage \& Panek (1974), with the sign convention used through out this work. Contamination of stellar Ly $\alpha$ by the interstellar H I absorption is a serious concern, in particular for O stars whose larger average distances (when compared to B stars) lead to interstellar neutral hydrogen column densities $N_{H}$ in excess of $10^{20} \mathrm{~cm}^{-2}$. As a result, the measured Ly $\alpha$ EW's in essentially all $\mathrm{O}$ stars are not stellar but interstellar. Since the derived lower limits of the stellar contribution to the total Ly $\alpha$ would provide little insight into the validity of our models, we did not consider stars with $T_{\text {eff }}>35,000 \mathrm{~K}$ for our comparison. This cut-off corresponds to spectral type O9. In Table 3 we present the selected stars together with the pertinent parameters. Columns 1 and 2 list the designations. The spectral types are in column $3 . T_{\text {eff }}$ (column 4) was assigned using the calibration of Conti et al. (2008). Observed EW values are in column 5.

In Figure 4 we show the comparison between the observed and theoretical EW values as a function of stellar effective temperature. The models display a near-monotonic trend with $T_{\text {eff }}$, asymptotically reaching about zero $\AA$ at $T_{\text {eff }}>35,000 \mathrm{~K}$. At a given $T_{\text {eff }}$, a larger $\log g$ results in stronger $\operatorname{Ly} \alpha$ absorption. The observed values track the models very well at the lowest temperatures but tend to be more negative (stronger absorption) at higher temperatures (corresponding to early B stars). We interpret this as not due to a model failure but rather being caused by residual interstellar contamination. In support of this suggestion we indicated the measured $E(B-V)$ values for each observation, taken from Savage \& Code (1970) and Savage \& Panek (1974). There is a clear trend of the largest discrepancies between models and data occurring for the highest $E(B-V)$ values. Since there is a well-established relation between $E(B-V)$ and interstellar $E(B-V)$ (Diplas \& Savage 1994a), we conclude that the majority of the observational points below the models are in fact just lower limits to the stellar contribution.

The outcome of the comparison between the modeled and observed EW's is quite reassuring. While there are few observational constraints on the Ly $\alpha \mathrm{EW}$ in $\mathrm{O}$ stars, the intrinsic Ly $\alpha$ in the hottest stars is expected to be weak because of the counteracting effects of photospheric absorption and wind emission. Therefore these stars would make only minor 
contribution to the total stellar absorption in a representative population. B stars, on the other hand, when present in a population can contribute substantially, and their modeled Ly $\alpha$ is in very good agreement with the data.

\section{Population Synthesis Models}

The Ly $\alpha$ EW's listed in Table 2 were implemented in the synthesis code Starburst99 (Leitherer et al. 1999; Leitherer \& Chen 2009). We performed a 2-dimensional interpolation in $T_{\text {eff }}$ and $\log g$ to obtain the EW values for each point in the Hertzsprung-Russell diagram as prescribed by the stellar evolutionary tracks. The input table is essentially complete at the highest temperatures, and no significant extrapolations were required. For $T_{\text {eff }}$ between 15,000 K and 10,000 K (late B stars) we used eq. (1) of Valls-Gabaud (1993) to approximate the Ly $\alpha$ EW. No attempts was made to account for EW at even lower $T_{e f f}$, and we simply assumed a featureless continuum for $T_{\text {eff }}<10,000 \mathrm{~K}$. This means our models are no longer valid when A stars begin to contribute to the UV continuum. We do not distinguish between O- and Wolf-Rayet stars when assigning Ly $\alpha$ EW's to stars in the Hertzsprung-Russell diagram. The underlying assumption is that Ly $\alpha$ (or the He II line at approximately the same wavelength) in Wolf-Rayet stars behaves in a similar fashion as in hot O stars.

The nebular Ly $\alpha$ was calculated assuming standard Case B recombination for an electron temperature of $10^{4} \mathrm{~K}$. For reference, the $\mathrm{Ly} \alpha / \mathrm{H} \alpha$ ratio is 8.7 under these assumptions (Case A would be 11.4).

We created a standard set of synthetic Ly $\alpha$ EW for evolving stellar populations using the Geneva evolution models with high mass loss at solar chemical composition (Meynet et al. 1994). As a consistency check, we compared our predictions with those published in the literature. A standard Salpeter initial mass function (IMF) has been adopted for these comparisons. Charlot \& Fall (1993) and Valls-Gabaud (1993) were the first to draw attention to the importance of underlying stellar Ly $\alpha$ for the interpretation of nebular Ly $\alpha$ in star-forming galaxies. They both used the original Bruzual \& Charlot (1993) evolutionary synthesis models with a correction of stellar Ly $\alpha$ to predict the net nebular + stellar Ly $\alpha$. The stars evolve along the tracks computed by Maeder \& Meynet (1989) in these models. In Table 4 we compare their and our predictions for selected ages and for the two limiting cases of an instantaneous burst and continuous star formation. Also included in Table 4 are

the predictions of Schaerer \& Verhamme (2008) who used a modified version of the Schaerer (2003) evolutionary synthesis models for predictions of nebular + stellar Ly $\alpha$. The different sets of models are in reasonable agreement. The differences reflect the use of different evolutionary tracks and different model atmospheres. Note in particular that the larger EW at 
age $5 \times 10^{6} \mathrm{yr}$ in the Charlot \& Fall and Valls-Gabaud models is the result of different life times in the stellar evolution models. At this age, the nebular Ly $\alpha$ displays a sharp drop with age, and a slight shift in age causes a vastly different ionizing photon output.

We performed a parameter study in order to analyze the impact of different IMFs, different ages, and different star-formation histories in the Ly $\alpha$ EWs. The two bracketing cases of an instantaneous burst and constant star formation were considered. Starburst99 assumes a power-law IMF of the classical form $\phi(m) \propto m^{-\alpha}$. In this notation, the classical Salpeter IMF has an exponent of $\alpha=2.35$. In Figures 5 and 6 we show the resulting Ly $\alpha$ EWs for different IMF slopes in the case of an instantaneous burst and continuous star formation, respectively.

We first address the relative importance of the underlying stellar Ly $\alpha$ at different ages in each of the two cases. The burst models (Fig. 5) fall into two regimes: at young ages (t $\lesssim 6 \mathrm{Myr}$ ), the nebular part always dominates since copious $\mathrm{O}$ stars photons are available and $\mathrm{O}$ stars have intrinsically weak Ly $\alpha$. At older ages ( $\mathrm{t} \gtrsim 10 \mathrm{Myr}$ ), the starburst is B-star dominated with few ionizing photons but at the same time intrinsically strong stellar Ly $\alpha$. In the case of continuous star formation (Figure 6), the nebular component always dominates, and the underlying stellar Ly $\alpha$ is always negligible. As a reminder, this assumes pure Case A recombination for the nebular Ly $\alpha$ with a $100 \%$ photon escape.

Different IMF exponents, ranging from an extreme, flat IMF of $\alpha=0.5$ to another extreme, steep value of $\alpha=2.6$, only have a minor influence in the stellar Ly $\alpha$ EWs. The equivalent width varies by about $2 \AA$ for the considered IMF exponents between 0.5 and 2.6 for an instantaneous burst. This is expected for a single population where the stellar flux at a particular wavelength comes from stars over a very narrow mass range. In the continuous case, the IMF sensitivity of the stellar Ly $\alpha$ is somewhat more pronounced but still quite minor.

Next, we turn to the IMF dependence of the nebular component of Ly $\alpha$. The IMF has a much more pronounced effect in this case, which can easily be understood from the fact the $\mathrm{EW}(\mathrm{Ly} \alpha)$ is determined by the ratio of early- to late-O stars, which contribute the ionizing photons and the continuum. Varying the IMF slope will change the relative contributions of these species. Therefore the IMF behavior in Figs. 5 and 6 is not surprising and mirrors that of, e.g., the $\mathrm{H} \alpha$ or $\mathrm{H} \beta$ equivalent width, which has been extensively discussed in the literature (Leitherer et al. 1999).

The previous discussion is based on the assumption of a $100 \%$ escape probability of the nebular Ly $\alpha$ photons. Observations suggest a much lower escape fraction. For instance the Ly $\alpha$ EWs observed in Lyman-break galaxies at redshift $\sim 3$ are of order $10 \AA$ (Shapley et al. 
2003). Comparison with our continuous models uncorrected for stellar absorption, implies an escape of Ly $\alpha$ photons of $\sim 10 \%$. In order to provide a more realistic model grid for comparison with data, we generated models with a Salpeter IMF and nebular Ly $\alpha$ escape fractions of 5, 10, 25, 50 and 100\%. These models are plotted in Figures 7 and 8 for instantaneous bursts and continuous star formation, respectively. Because of the reduced nebular emission for escape fractions less than 100\%, the underlying stellar absorption drastically increases in its contribution to the net EW. For instance, if the escape fraction is $10 \%$ the stellar absorption and nebular emission EW's become comparable for continuous star formation at ages of 10 to $20 \mathrm{Myr}$.

\section{Conclusions}

We present a grid of theoretical stellar Ly $\alpha$ equivalent widths for evolving stellar populations. While these models have value in their own right, our main motivation for presenting the grid is the need to correct nebular Ly $\alpha$ emission in star-forming galaxies for underlying stellar absorption. This study builds on the pioneering work of Valls-Gabaud (1993) and Charlot \& Fall (1993) who had to rely on earlier, less complete model atmospheres, and it extends the exploratory work of Schaerer \& Verhamme (2008), who used the latest model atmospheres for a restricted parameter range.

We compiled a new library of non-LTE Ly $\alpha$ EWs based on fully line-blanketed models. The library includes $\mathrm{O}$ and $\mathrm{B}$ stars with effective temperatures ranging from $15,000 \mathrm{~K}$ to 48,500 K, and $\log g$ from 1.75 to 4.5. The measured Ly $\alpha$ EW's range from 5.9 to $-32.5 \AA$. In particular, we obtained: Ly $\alpha$ EW between 0.9 and $-32.5 \AA$ for effective temperatures of $15,000 \mathrm{~K}$ to $29,000 \mathrm{~K}$, and values mostly close to $0 \AA$ for higher effective temperatures due to the canceling effects of the emission and absorption of the P Cygni profiles of these stars. Metallicity has very little effect on the Ly $\alpha$ EW's.

We implemented the library in Starburst99 and studied the behavior of the Ly $\alpha$ EW for a range of stellar population properties. We performed a parameter study of instantaneous and continuous star formation at solar metallicity, using the high-mass loss Geneva evolution models (Meynet et al. 1994). We found: (i) The IMF has only minor influence on the stellar EW when varied within astrophysically plausible limits for both an instantaneous and a constant SFR. (ii) The IMF has also a minor influence on the nebular component of the Ly $\alpha$ EW for an instantaneous SFR, however, for a continuous SFR, the difference between the two extreme IMF exponents (0.5 and 2.6) in the nebular component of the Ly $\alpha \mathrm{EW}$ is about $100 \AA$ at $3 \mathrm{Myr}$ and about $180 \AA$ at $15 \mathrm{Myr}$. (iii) When $\mathrm{O}$ stars dominate the spectrum (as indicated by the presence of nebular emission lines), stellar Ly $\alpha$ absorption is always 


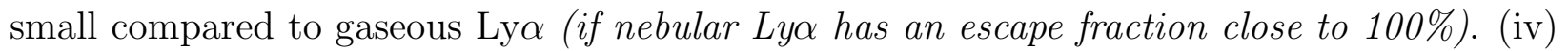
Depending on the escape fraction of nebular Ly $\alpha$ photons, the stellar contribution to the total ranges from negligible to dominant. If the nebular escape fraction is $10 \%$, the stellar absorption and nebular emission equivalent widths become comparable for continuous star formation at ages of 10 to $20 \mathrm{Myr}$.

In stark contrast to the often used a stellar absorption correction of 50 to $240 \AA$, our models predict: (i) stellar Ly $\alpha$ EW values of 9 to $18 \AA$ in absorption for an instantaneous burst between the ages of 5 to $15 \mathrm{Myr}$, and (ii) values of 2 to $8 \AA$ also in absorption for constant star formation in the same age range. Our models provide a realistic description of the stellar Ly $\alpha$ and should be appropriate to correct the nebular emission for underlying stellar absorption in star-forming galaxies.

AKNOWLEDGEMENTS. We are grateful to an anonymous referee for a careful reading of the manuscript and many useful suggestions. We are also grateful to John Hillier for kindly providing us with plane-parallel CMFGEN models to check consistency between the results of CMFGEN and TLUSTY modeled atmospheres. Support for this work has been provided by NASA through grant number N-1317 from the Space Telescope Science Institute, which is operated by AURA, Inc., under NASA contract NAS5-26555.

\section{REFERENCES}

Bohlin, R. C., Savage, B. D., \& Drake, J. F. 1978, ApJ, 224, 132

Bromm, V. \& Yoshida, N. 2011, A\&A, 49, 373

Bruzual, G. \& Charlot, S. 1993, ApJ, 405, 538

Charlot, S. \& Fall, M. 1993, ApJ, 415, 580

Conti, P. S., Crowther, P. A., \& Leitherer, C. 2008, "From Luminous Hot Stars to Starburst Galaxies", Cambridge University Press, Cambridge, UK

Dijkastra, M. \& Wyithe, S. B. 2010, MNRAS, 408, 352

Dijkastra, M. \& Wyithe, S. B. 2012, MNRAS, 419, 3181

Diplas, A. \& Savage, B. 1994a, ApJ, 427, 274

Diplas, A. \& Savage, B. 1994b, ApJS, 93, 211 
Fujita, S. S., Ajiki, M., Shioya, Y., et al. 2003, AJ, 125, 13

Giavalisco, M., Koratkar, A., \& Calzetti, D. 1996, ApJ, 446, 831

González-Delgado, R. M. \& Leitherer, C. 1999, ApJ, 125, 479

Haiman, Z. \& Spaans, M. 1999, AIPC, 470, 63

Hansen, M. \& Oh, S. P. 2006, MNRAS, 367, 979

Hartmann, L. W., Huchra, J. P., Geller, M. J., O’Brien, P., \& Wilson, R. 1988, ApJ, 326, 101

Heap, S. R., Lanz, T., \& Hubeny, I. 2006, ApJ, 638, 409

Hillier, D. J. 1987, ApJS, 63, 947

Hillier, D. J. 2011. Hot Stars with Winds: The CMFGen Code. Proceedings of the International Astronomical Union, 7, pp 229-234. doi:10.1017/S1743921311027426.

Hillier, D. J. \& Lanz, T. 2001, ASPC, 247, 343

Hillier, D. J. \& Miller, D. L. 1998, ApJ, 496, 407

Hubeny, I., \& Lanz, T., 1995, AJ, 439, 875

Kennicutt, R. C. \& Evans, N. J. 2012, ARA\&A, 50, 531

Klein, R. I. \& Castor, J. I. 1978, ApJ, 220, 902

Kunth, D., Leitherer, C., Mas-Hesse, J. M., 'Ostlin, G., \& Petrosian, A. 2003, ApJ, 597, 263

Lallement, R., Quémarais, E., Bertaux, J. L., et al. 2011. Science Magazzine, Vol. 334, www.sciencemag.org

Lamers, H. J. G. L. M., \& Cassinelli, J. P. 1999, Introduction to Stellar Winds, ed. H. J. G. L. M. Lamers \& J. P. Cassinelli

Lanz, T. \& Hubeny, I. 2003, ApJS, 147, 225

Lanz, T. \& Hubeny, I. 2007, ApJS, 169, 83

Laursen, P., Duval, F., \& Östlin, G. 2013, ApJ, 766, 124

Leitherer, C. \& Chen, J. 2009, New A. 4, 356 
Leitherer, C., Ortiz, P., Bresolin, F., et al. 2010, ApJSS, 189, 309

Leitherer, C., Robert, C., \& Heckman T. M. 1995, 99, 173

Leitherer, C., Schaerer, D., Goldader, J., et al. 1999, ApJ, 123, 3

Maeder, A., \& Meynet, G. 1989, A\&A, 210, 155

Malhotra, S. \& Rhoads, J. 2002, ApJ, 565, L71

Malhotra, S. \& Rhoads, J. 2004, ApJ, 617, L5

Mas-Hesse, J. M., Kunth, D., Tenorio-Tagle, G., Leitherer, C., Terlevich, R. J., \& Terlevich, E. 2003, ApJ, 598, 858

Massey, P., Neugent, K. F., Hillier, J. D., \& Puls, J. 2013, to be published in AJ, arXiv: $1303.5469 \mathrm{v} 1$

Meier, D. L. \& Terlevich, R. 1981, ApJ, 247L, 109

Meynet, G., Maeder, A., Schaeller, G., et al. 1994, A\&A, 103, 97

Neufeld, D. 1990, ApJ, 350, 216

Palacios, A., Gebran, M., Josselin, E., et al. 2010, A\&A, 516, 13

Partridge, R. B. \& Peebles, P. J. E. 1967, ApJ, 147, 868

Puls, J., Vink, J. S., \& Najarro, F. 2008, A\&A, 16, 209

Sandberg, A., Östlin, G., Hayes, M., et al. 2013, A\&A, 552, id.A95

Savage, B. D. \& Code, A. D. 1970, in Ultraviolet Stellar Spectra and Related Ground-based Observations (IAU Symposium No. 36) ed. Houziaux, L. and Butler, H. E. (Dordrecht: D. Reidel Publishing Co.), p. 302

Savage, B. D. \& Jenkins, E. B. 1972, ApJ, 172, 491

Savage, B. D. \& Panek, R. J. 1974, ApJ, 191, 659

Scarlata, C, Colbert, J., Teplitz, H. I., et al. 2009, ApJ, 704, L98

Shapley, A. E., Steidel, C. C., Pettini, M., \& Adelberger, K. L. 2003, ApJ, 588, 65

Schaerer, D. 2003, A\&A, 397, 527 
Schaerer, D. \& Verhamme, A. 2008, A\&A, 480, 369

Stern, D., Yost, S. A., Eckart, M. E., et al. 2005, ApJ, 619, 12

Valls-Gabaud, D. 1993, ApJ, 419, 7

Vink, J. S. 2007, AIPC, 948, 389

Walborn, N. R., Nichols-Bohlin, J., \& Panek, R. J. 1985, International Ultraviolet Explorer Atlas of O-Type Spectra from 1200 to $1900 \AA$ (NASA RP-1155)

Walborn, N. R., Parker, J. W., \& Nichols-Bohlin, J. 1995, International Ultraviolet Explorer Atlas of B-Type Spectra from 1200 to 1900 Å (NASA RP-1363) 
Table 1. Parameters of the models used for the new library.

\begin{tabular}{|c|c|c|}
\hline$T_{\text {eff }}[\mathrm{K}]$ & $\log g$ & Code \\
\hline 15,000 & $1.75,2.00,2.25,2.50,2.75,3.00,3.25,3.50,3.75,4.00,4.25,4.50$ & TLUSTY \\
\hline 16,000 & $2.00,2.25,2.50,2.75,3.00,3.25,3.50,3.75,4.00,4.25,4.50$ & TLUSTY \\
\hline 17,000 & $2.00,2.25,2.50,2.75,3.00,3.25,3.50,3.75,4.00,4.25,4.50$ & TLUSTY \\
\hline 18,000 & $2.00,2.25,2.50,3.00,3.25,3.50,3.75,4.00,4.25,4.50$ & TLUSTY \\
\hline 19,000 & $2.25,2.50,2.75,3.00,3.25,3.50,3.75,4.00,4.25,4.50$ & TLUSTY \\
\hline 20,000 & $2.25,2.50,2.75,3.00,3.25,3.50,3.75,4.00,4.25,4.50$ & TLUSTY \\
\hline 21,000 & $2.50,2.75,3.00,3.25,3.50,3.75,4.00,4.25,4.50$ & TLUSTY \\
\hline 22,000 & $2.50,2.75,3.00,3.25,3.50,3.75,4.00,4.25,4.50$ & TLUSTY \\
\hline 23,000 & $2.50,2.75,3.00,3.25,3.50,3.75,4.00,4.25,4.50$ & TLUSTY \\
\hline 24,000 & $2.50,2.75,3.00,3.25,3.50,3.75,4.00,4.25,4.50$ & TLUSTY \\
\hline 25,000 & $2.50,2.75,3.00,3.25,3.50,3.75,4.00,4.25,4.50$ & TLUSTY \\
\hline 26,000 & $2.75,3.00,3.25,3.50,3.75,4.00,4.25,4.50$ & TLUSTY \\
\hline 27,000 & $2.75,3.00,3.25,3.50,3.75,4.00,4.25,4.50$ & TLUSTY \\
\hline 27,500 & $3.00,3.75$ & CMFGEN \\
\hline 27,730 & 3.35 & CMFGEN \\
\hline 28,000 & $2.75,3.00,3.25,3.50,3.75,4.00,4.25,4.50$ & TLUSTY \\
\hline 29,000 & $3.00,3.25,3.50,3.75,4.00,4.25,4.50$ & TLUSTY \\
\hline 30,000 & $3.00,3.25,3.50,3.75,4.00,4.25,4.50$ & TLUSTY \\
\hline 30,000 & $3.00,3.13,3.50,4.00,4.10,4.20$ & CMFGEN \\
\hline 30,270 & 3.29 & CMFGEN \\
\hline 30,410 & 3.73 & CMFGEN \\
\hline 31,480 & 4.06 & CMFGEN \\
\hline 32,210 & 3.26 & CMFGEN \\
\hline 32,500 & $3.25,3.50,3.60,4.10,4.25$ & CMFGEN \\
\hline 32,660 & 3.71 & CMFGEN \\
\hline 33,340 & 4.01 & CMFGEN \\
\hline 34,435 & 3.52 & CMFGEN \\
\hline 35,000 & $3.25,3.50,4.10,4.25$ & CMFGEN \\
\hline 36,310 & 4.02 & CMFGEN \\
\hline 37,411 & 3.38 & CMFGEN \\
\hline 37,500 & $3.50,3.90$ & CMFGEN \\
\hline 37,670 & 3.77 & CMFGEN \\
\hline 37,760 & 3.76 & CMFGEN \\
\hline 39,540 & 3.68 & CMFGEN \\
\hline 39,628 & 3.93 & CMFGEN \\
\hline 39,994 & 3.63 & CMFGEN \\
\hline 40,000 & $3.50,3.90$ & CMFGEN \\
\hline 41,020 & 4.04 & CMFGEN \\
\hline 41,591 & 3.80 & CMFGEN \\
\hline 41,178 & 4.02 & CMFGEN \\
\hline 42,560 & 3.71 & CMFGEN \\
\hline 42,560 & 4.16 & CMFGEN \\
\hline 43,954 & 3.98 & CMFGEN \\
\hline 46,130 & 4.05 & CMFGEN \\
\hline 48,530 & 4.01 & CMFGEN \\
\hline
\end{tabular}


Table 2. Ly $\alpha$ Equivalent Widths* as a function of Effective Temperature and $\log g$.

\begin{tabular}{|c|c|c|c|c|c|c|c|c|c|c|c|c|}
\hline \multirow{2}{*}{$T_{e f f}[\mathrm{~K}]$} & \multicolumn{12}{|c|}{ Ly $\alpha$ EW $[\AA]$ for $\log g$ of: } \\
\hline & 1.75 & 2.00 & 2.25 & 2.50 & 2.75 & 3.00 & 3.25 & 3.50 & 3.75 & 4.00 & 4.25 & 4.50 \\
\hline 15,000 & -15.3 & -20.6 & -18.3 & -22.6 & -25.5 & -25.6 & -24.6 & -27.9 & -29.3 & -29.3 & -32.6 & -32.5 \\
\hline 16,000 & -12.2 & -13.8 & -16.8 & -17.8 & -19.8 & -20.8 & -23.7 & -24.2 & -25.0 & -27.5 & 28.8 & -29.7 \\
\hline 17,000 & -9.6 & -11.1 & -18.4 & -16.0 & -16.6 & -19.7 & -21.7 & -21.7 & -23.8 & -24.6 & -26.9 & -25.9 \\
\hline 18,000 & -3.8 & -5.7 & -11.4 & -13.6 & -15.5 & -15.4 & -17.1 & -19.4 & -20.3 & -24.4 & -22.2 & -24.3 \\
\hline 19,000 & -5.2 & -7.0 & -8.7 & -9.4 & -15.7 & -14.3 & -18.5 & -18.6 & -20.6 & -19.8 & -20.7 & -24.1 \\
\hline 20,000 & -5.8 & -7.2 & -8.7 & -11.5 & -13.6 & -13.6 & -16.1 & -15.5 & -18.01 & -17.9 & -20.8 & -21.7 \\
\hline 21,000 & -8.1 & -9.2 & -10.4 & -11.6 & -10.8 & -12.5 & -14.5 & -14.4 & -16.9 & -17.9 & -18.8 & -20.9 \\
\hline 22,000 & -5.0 & -6.3 & -7.5 & -8.7 & -10.6 & -12.2 & -12.4 & -14.9 & -15.2 & -16.9 & -18.8 & -18.6 \\
\hline 23,000 & -1.3 & -2.7 & -4.0 & -5.4 & -10.9 & -11.0 & -12.3 & -12.2 & -14.8 & -14.3 & -19.2 & -16.2 \\
\hline 24,000 & 0.9 & -0.9 & -2.7 & -4.5 & -9.0 & -10.0 & -12.4 & -13.5 & -15.7 & -17.0 & -15.8 & -18.8 \\
\hline 25,000 & -0.7 & -2.2 & -3.7 & -5.3 & -6.8 & -11.2 & -11.4 & -11.9 & -12.7 & -16.3 & -16.0 & -17.5 \\
\hline 26,000 & -4.8 & -5.8 & -6.8 & -7.8 & -8.8 & -9.6 & -10.6 & -10.7 & -14.1 & -12.9 & -14.5 & 15.9 \\
\hline 27,000 & -3.5 & -4.4 & -5.3 & -6.2 & -7.0 & -10.4 & -8.5 & -10.0 & -12.2 & -11.8 & -14.0 & -13.2 \\
\hline 28,000 & -1.0 & -2.0 & -3.1 & -4.2 & -5.2 & -7.5 & -6.6 & -8.1 & -11.2 & -12.8 & -11.1 & -12.7 \\
\hline 29,000 & -3.5 & -4.2 & -4.9 & -5.6 & -6.3 & -7.0 & -6.0 & -8.0 & -11.0 & -12.0 & -11.0 & -11.2 \\
\hline 30,000 & 0.4 & -0.1 & -0.5 & -0.9 & -1.3 & -1.7 & -2.0 & -2.6 & -3.0 & -3.4 & -3.8 & -4.3 \\
\hline 32,500 & 1.5 & 1.0 & 0.5 & -0.0 & -0.5 & -1.1 & -1.4 & -2.1 & -2.4 & -3.1 & -2.6 & -4.1 \\
\hline 35,000 & 5.9 & 5.0 & 4.0 & 3.1 & 2.1 & 1.2 & 0.2 & -1.3 & -1.7 & -2.6 & -2.8 & -4.5 \\
\hline 37,500 & 0.5 & 0.4 & 0.3 & 0.1 & 0.0 & -0.1 & -0.2 & -0.4 & -0.5 & -0.6 & -0.7 & -0.9 \\
\hline 40,000 & 2.0 & 1.7 & 1.4 & 1.1 & 0.8 & 0.5 & 0.2 & -0.1 & -0.4 & -0.7 & -1.0 & -1.3 \\
\hline 42,500 & 2.2 & 1.8 & 1.5 & 1.2 & 0.9 & 0.5 & 0.3 & -0.1 & -0.4 & -0.7 & -1.0 & -1.4 \\
\hline 45,000 & 0.7 & 0.6 & 0.4 & 0.2 & 0.1 & -0.1 & -0.2 & -0.4 & -0.5 & -0.7 & -0.9 & -1.0 \\
\hline 48,500 & -0.3 & -0.4 & -0.4 & -0.5 & -0.6 & -0.6 & -0.7 & -0.7 & -0.8 & -0.9 & -0.9 & -1.0 \\
\hline
\end{tabular}

${ }^{*}$ The sign convention used for this work is positive for emission and negative for absorption. 
Table 3. Ly $\alpha$ Equivalent Widths Measurements from Observations* as a function of Effective Temperature.

\begin{tabular}{|c|c|c|c|c|}
\hline $\begin{array}{c}\text { Star } \\
{[\text { HD number }]}\end{array}$ & Name & Spectral Type & Ly $\alpha \mathrm{EW}[\AA]$ & $T_{e f f}[\mathrm{~K}]$ \\
\hline \multicolumn{5}{|c|}{ Taken from Savaqe \& Code $(1970)^{\mathrm{a}}$} \\
\hline $11415^{\mathrm{b}}$ & $\epsilon$ Cas & B3 IVp & -28 & 17,500 \\
\hline 24398 & $\zeta$ Per & B1 Ib & -25 & 21,500 \\
\hline 24760 & $\epsilon$ Per & $\mathrm{B} 0.5 \mathrm{~V}$ & -14 & 28,000 \\
\hline 29763 & $\tau$ Tau & B3 V & -29 & 17,500 \\
\hline $32630^{\mathrm{b}}$ & $\eta \mathrm{Au}$ & B3 V & -25 & 17,500 \\
\hline 34816 & $\lambda$ Lep & B0.5 IV & -14 & 27,500 \\
\hline 35411 & $\eta$ Ori & $\mathrm{B} 0.5 \mathrm{~V}$ & -20 & 28,000 \\
\hline 35439 & 25 Ori & B1 Vpe & -19 & 26,000 \\
\hline 35468 & $\gamma$ Ori & B2 III & -15 & 19,750 \\
\hline $35497^{\mathrm{b}}$ & $\beta \mathrm{Tau}$ & B7 III & -35 & 12,700 \\
\hline $36485-86$ & $\delta$ Ori & O9.5 II & -20 & 29,625 \\
\hline 36512 & $\nu$ Ori & B0 V & -13 & 29,500 \\
\hline 36822 & $\phi^{\prime}$ Ori & B0 IV & -31 & 29,000 \\
\hline 37022 & 41 Ori & $\mathrm{O} 7 \mathrm{~V}$ & & 37,000 \\
\hline-41 & $\theta$ Ori & O9.5 Vp & -33 & 31,500 \\
\hline 37043 & $\iota$ Ori & O9 III & -17 & 32,250 \\
\hline 37128 & $\epsilon$ Ori & B0 Ia & -24 & 27,500 \\
\hline 37202 & $\zeta \mathrm{Tau}$ & B2 IVp & -21 & 20,375 \\
\hline 37468 & $\sigma$ Ori & O9.5 V & -16 & 31,500 \\
\hline $37742-43$ & $\zeta$ Ori & O9.5 Ib & -25 & 29,000 \\
\hline 38771 & $\kappa$ Ori & B0.5 Ia & -22 & 26,000 \\
\hline 44743 & $\beta \mathrm{CMa}$ & B1 II & -10 & 22,625 \\
\hline 52089 & $\epsilon \mathrm{CMa}$ & B2 II & -12 & 19,125 \\
\hline $87901^{b}$ & $\alpha$ Leo & B7 V & -43 & 13,300 \\
\hline 116658 & $\alpha \mathrm{Vir}$ & $\mathrm{B} 1 \mathrm{~V}$ & -11 & 26,000 \\
\hline $120315^{\mathrm{b}}$ & $\eta \mathrm{Uma}$ & B3 V & -22 & 17,500 \\
\hline 122451 & $\beta$ Cen & B1 II & -12 & 22,625 \\
\hline 127381 & $\sigma$ Lup & $\mathrm{B} 2 \mathrm{~V}$ & -20 & 21,000 \\
\hline 127972 & $\eta$ Cen & B1.5 Vne & -15 & 24,000 \\
\hline $128345^{\mathrm{b}}$ & $\rho \operatorname{Lup}$ & B5 V & -36 & 15,400 \\
\hline 129056 & $\alpha \operatorname{Lup}$ & B1 V & -14 & 26,000 \\
\hline
\end{tabular}


Table 3-Continued

\begin{tabular}{|c|c|c|c|c|}
\hline $\begin{array}{c}\text { Star } \\
{[\mathrm{HD} \text { number }]}\end{array}$ & Name & Spectral Type & Ly $\alpha \operatorname{EW}[\AA]$ & $T_{\text {eff }}[\mathrm{K}]$ \\
\hline 132200 & $\kappa$ Cen & $\mathrm{B} 2 \mathrm{~V}$ & -17 & 21,000 \\
\hline $133242-43^{\mathrm{b}}$ & $\pi$ Lup & B5 IV & -26 & 14,925 \\
\hline $133955^{\mathrm{b}}$ & $\lambda \operatorname{Lup}$ & B3 V & -22 & 17,500 \\
\hline 136298 & $\delta \operatorname{Lup}$ & B2 IV & -14 & 20,375 \\
\hline 136664 & $\phi^{2} \operatorname{Lup}$ & B5 V & -26 & 15,400 \\
\hline $139365^{\mathrm{b}}$ & $\tau \mathrm{Lib}$ & $\mathrm{B} 2.5 \mathrm{~V}$ & -22 & 19,000 \\
\hline 141637 & $1 \mathrm{Sco}$ & $\mathrm{B} 2.5 \mathrm{Vn}$ & -30 & 19,000 \\
\hline 142983 & $48 \mathrm{Lib}$ & B8 Ia/Iab & -12 & 11,400 \\
\hline 143018 & $\pi \mathrm{Sco}$ & $\mathrm{B} 1 \mathrm{~V}$ & -18 & 26,000 \\
\hline 143275 & $\delta \mathrm{Sco}$ & B0 V & -24 & 29,500 \\
\hline 144470 & $\omega^{1} \mathrm{Sco}$ & $\mathrm{B} 1 \mathrm{~V}$ & -33 & 26,000 \\
\hline 147165 & $\sigma \mathrm{Sco}$ & B1 III & -31 & 23,750 \\
\hline 149757 & $\zeta \mathrm{Oph}$ & $09.5 \mathrm{~V}$ & -26 & 31,500 \\
\hline 151890 & $\mu^{\prime}$ Sco & $\mathrm{B} 1.5 \mathrm{~V}$ & -20 & 24,000 \\
\hline 158926 & $\lambda^{\prime}$ Sco & B1 V & -12 & 26,000 \\
\hline 160578 & $\kappa \mathrm{Sco}$ & B2 IV & -16 & 20,375 \\
\hline 189103 & $\Theta^{\prime} \operatorname{Sgr}$ & B3 IV & -29 & 17,000 \\
\hline $209952^{\mathrm{b}}$ & $\alpha$ Gru & B5 V & -33 & 15,400 \\
\hline \multicolumn{5}{|c|}{ Taken from Savaqe $\& 3$ Panek $(1974)^{c}$} \\
\hline 358 & $\alpha$ And & B9 II & -45 & 10,850 \\
\hline 10144 & $\alpha$ Eri & B3 Vp & -21 & 17,500 \\
\hline $11415^{\mathrm{b}}$ & $\epsilon \mathrm{Cas}$ & $\mathrm{B} 3 \mathrm{Vp}$ & -25 & 17,500 \\
\hline 19356 & $\beta$ Per & B8 V & -65 & 12,300 \\
\hline 22928 & $\delta$ Per & B5 III & -31 & 14,450 \\
\hline $32630^{\mathrm{b}}$ & $\eta$ Aur & B3 V & -22 & 17,500 \\
\hline 34085 & $\beta$ Ori & B8 Ia & $>-30$ & 11,400 \\
\hline $35497^{\mathrm{b}}$ & $\beta \mathrm{Tau}$ & B7 III & -42 & 12,700 \\
\hline 37795 & $\alpha \mathrm{Col}$ & B7 IV & -45 & 13,000 \\
\hline 42560 & $\xi$ Ori & B3 IV & -21 & 17,000 \\
\hline 44402 & $\zeta \mathrm{CMa}$ & B2.5 IV & -20 & 18,375 \\
\hline 6575 & $\chi \mathrm{Car}$ & B3 IVp & -19 & 17,000 \\
\hline $87901^{b}$ & $\alpha$ Leo & B7 V & -55 & 13,300 \\
\hline
\end{tabular}


Table 3-Continued

\begin{tabular}{lllcl}
\hline \hline $\begin{array}{c}\text { Star } \\
\text { HD number }]\end{array}$ & Name & Spectral Type & Ly $\alpha$ EW $[\AA]$ & $T_{e f f}[\mathrm{~K}]$ \\
\hline $120315^{\mathrm{b}}$ & $\eta$ UMa & B3 V & -25 & 17,500 \\
121263 & $\zeta$ Cen & B2.5 IV & -10 & 18,375 \\
125238 & $\iota$ Lup & B2.5 IV & -18 & 18,375 \\
125823 & $\alpha$ Cen & B7 IIIp & -23 & 12,700 \\
$128345^{\mathrm{b}}$ & $\rho$ Lup & B5 V & -45 & 15,400 \\
129116 & & B3 V & -20 & 17,500 \\
$133242^{\mathrm{b}}$ & $\pi$ Lup & B5 V & -30 & 15,400 \\
$-43^{\mathrm{c}}$ & & B5 IV & & 14,975 \\
$133955^{\mathrm{b}}$ & $\lambda$ Lup & B3 V & -21 & 17,500 \\
$139365^{\mathrm{b}}$ & $\tau$ Lib & B2.5 V & -19 & 19,000 \\
143118 & $\eta$ Lup & B2.5 IV & -11 & 18,375 \\
147394 & $\tau$ Her & B5 IV & -35 & 14,925 \\
155763 & $\zeta$ Dra & B6 III & -44 & 13,600 \\
160762 & $\iota$ Her & B3 IV & -21 & 17,000 \\
175191 & $\sigma$ Sgr & B3 IV & -18 & 17,000 \\
193924 & $\alpha$ Pav & B2.5 V & -17 & 19,000 \\
$209952^{\mathrm{b}}$ & $\alpha$ Gru & B7 IV & -45 & 13,000 \\
\hline
\end{tabular}

*The signs of the measured EW's were changed from original papers in order to maintain the convention of this work: absorption represented by a negative EW.

aThe EW's in this table include the blending with neighboring lines within $15 \AA$ (the OAO resolution), and also the blend with the interstellar $\operatorname{Ly} \alpha$ line.

${ }^{\mathrm{b}}$ These stars are present in both papers, Savage \& Code (1970) and Savage \& Panek (1974).

'The Savage \& Panek (1974) EW's in this table were those corrected for interstellar absorption and for the blend with neighboring lines. 
Table 4. Comparison of Ly $\alpha$ Equivalent Widths* . These values where determined for a Salpeter IMF, a stellar population with ages $5 \times 10^{6}$ and $15 \times 10^{6}$ years, and solar metallicity.

\begin{tabular}{|c|c|c|c|c|c|c|}
\hline \multirow[t]{2}{*}{ Authors } & \multicolumn{2}{|c|}{ Stellar component EW $[\AA]$} & \multicolumn{2}{|c|}{ Nebular component EW $[\AA]$} & \multicolumn{2}{|c|}{ Total EW $[\AA]=$ stellar + nebular } \\
\hline & $5 \times 10^{6}$ years & $15 \times 10^{6}$ years & $5 \times 10^{6}$ years & $15 \times 10^{6}$ years & $5 \times 10^{6}$ years & $15 \times 10^{6}$ years \\
\hline \multicolumn{7}{|l|}{ Instantaneous SFR } \\
\hline This work & -8 & -17 & 40 & 1 & 32 & -16 \\
\hline Charlot \& Fall (1993) & $\cdots$ & $\cdots$ & $\cdots$ & $\cdots$ & 210 & 53 \\
\hline Valls-Gabaud (1993) & -5 & -15 & 205 & 5 & 200 & -10 \\
\hline Schaerer \& Verhamme (2008) & -4 & -20 & 47 & 3 & 43 & -17 \\
\hline \multicolumn{7}{|l|}{ Constant SFR } \\
\hline This work & -4 & -7 & 145 & 100 & 141 & 93 \\
\hline Charlot \& Fall (1993) & $\cdots$ & $\cdots$ & $\ldots$ & $\ldots$ & 217 & 110 \\
\hline Valls-Gabaud (1993) & -3 & -5 & 253 & 195 & 250 & 190 \\
\hline Schaerer \& Verhamme (2008) & -1 & -4 & 131 & 80 & 130 & 76 \\
\hline
\end{tabular}

*The sign convention used for this work is positive for emission and negative for absorption. 


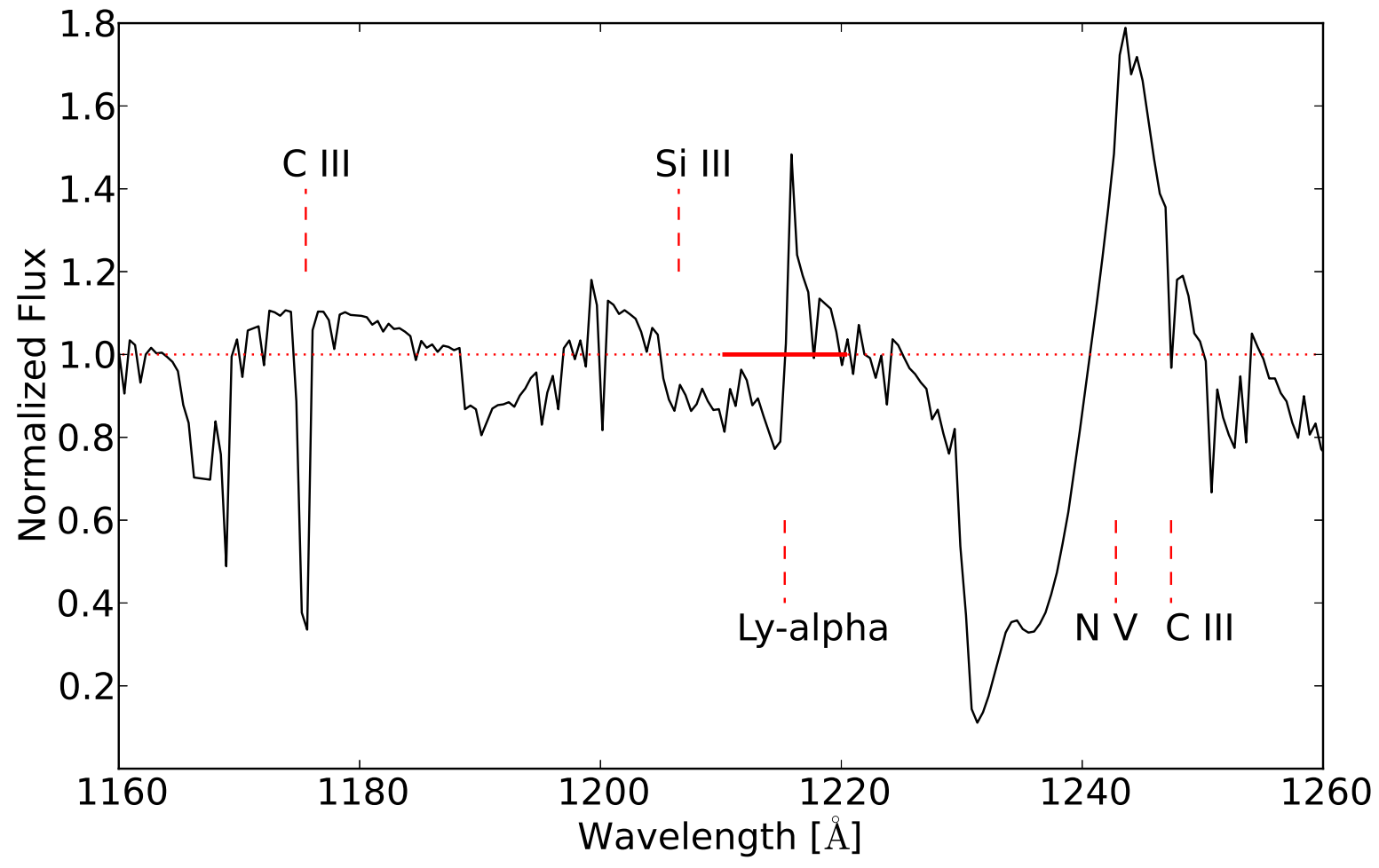

Fig. 1. - Theoretical spectrum of a CMFGEN O star with effective temperature of 40,000 $\mathrm{K}, \log g$ of 3.5 , and micro turbulent velocity of $10 \mathrm{~km} / \mathrm{s}$. The red dashed vertical lines mark the rest wavelength of the most prominent lines. The red horizontal dotted and solid lines mark the continuum and integration window used for the "simple integration" method (see Section 21), respectively. 


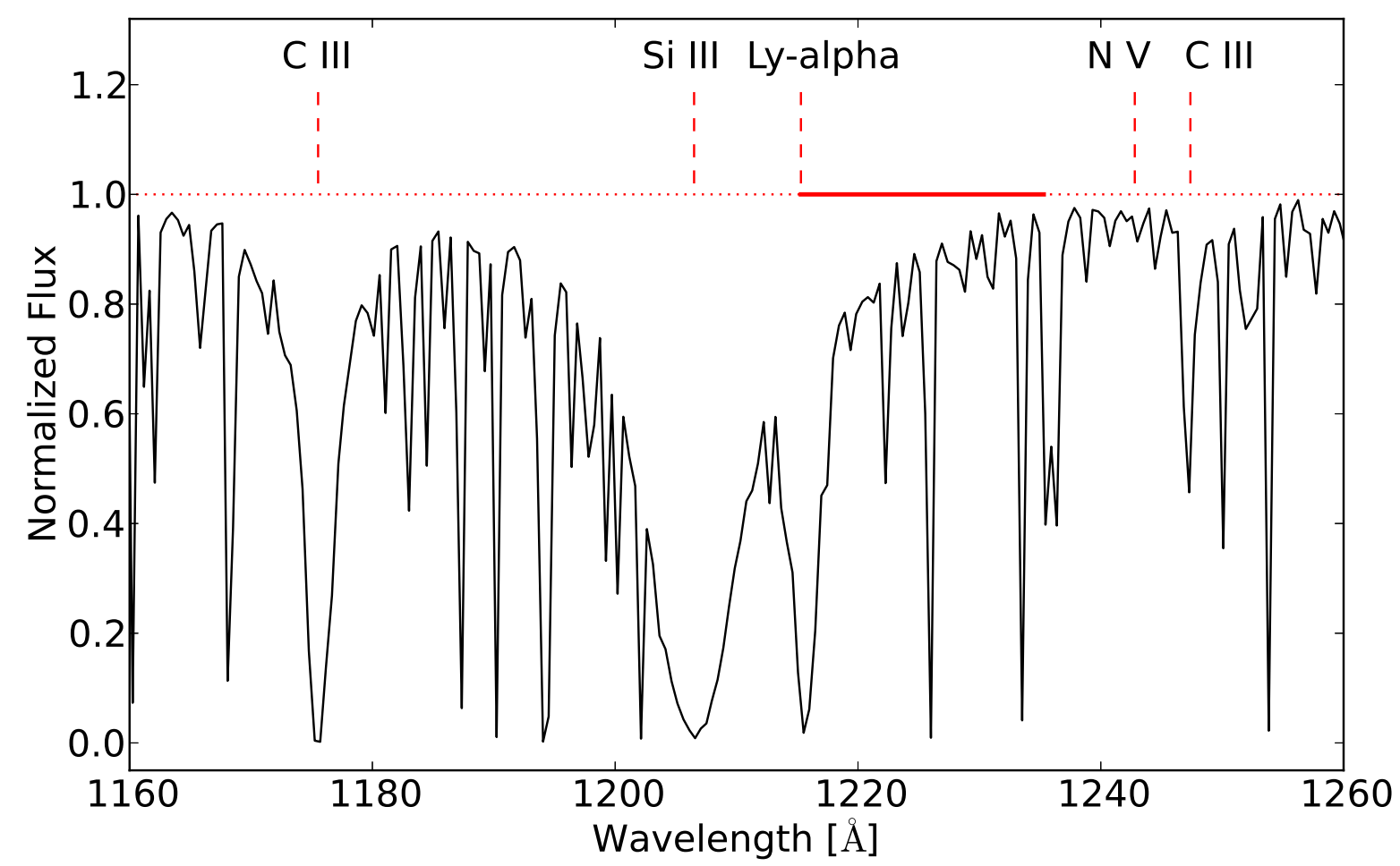

Fig. 2.- Theoretical spectrum of a TLUSTY B star with effective temperature of 18,000 K, $\log g$ of 4.25 , and micro turbulent velocity of $2 \mathrm{~km} / \mathrm{s}$. The red dashed vertical lines mark the rest wavelength of the most prominent lines. The red horizontal dotted and solid lines mark the continuum and integration window used for the "half integration" method (see Section 2), respectively. 


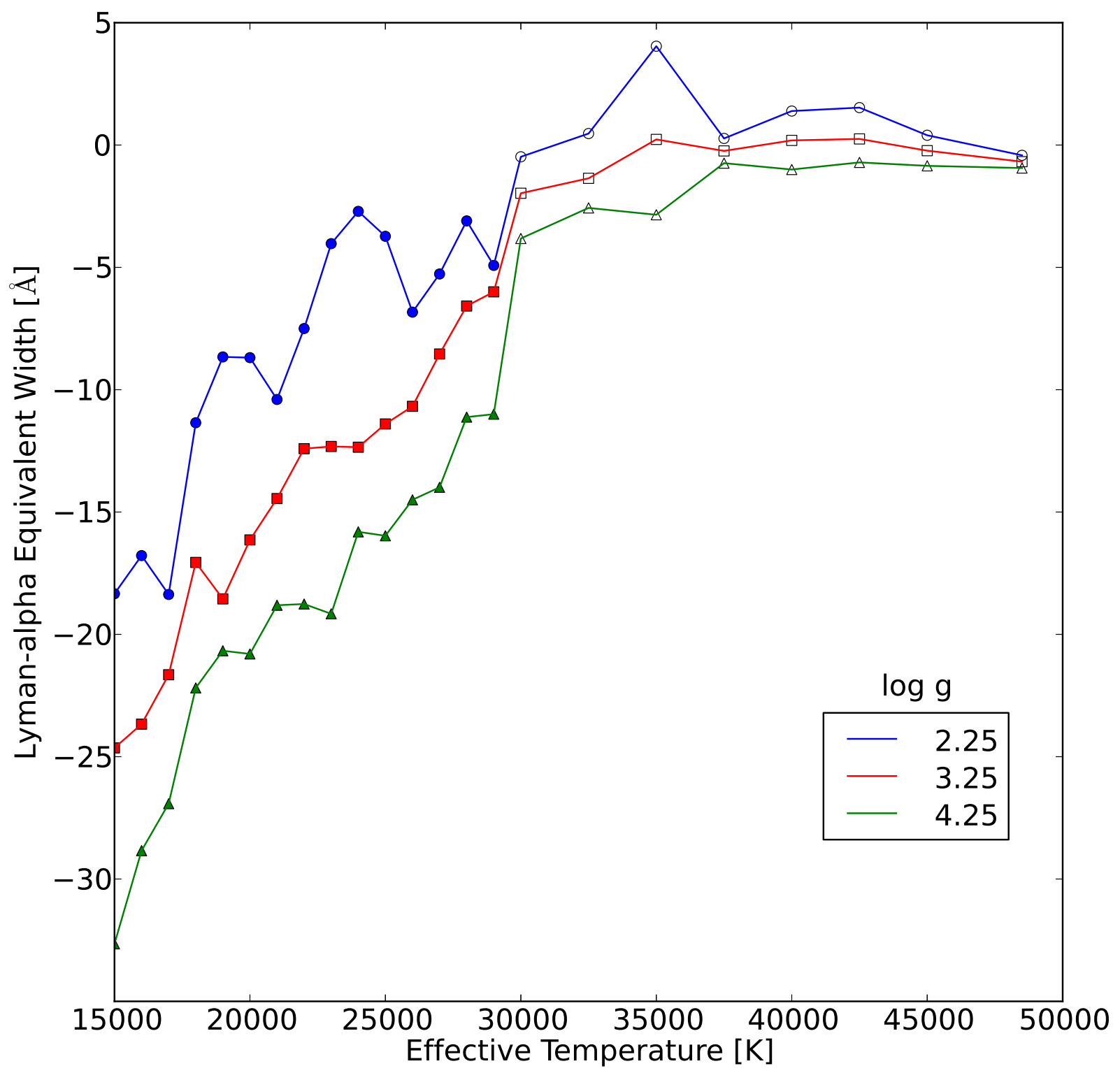

Fig. 3.- Plot of Ly $\alpha$ EW versus effective temperature. Three representative curves are presented. The top curve (blue circles) represents the Ly $\alpha$ EW for stars with a $\log g$ of 2.25 , the middle curve (red squares) for stars with a $\log g$ of 3.25 , and the lower curve (green triangles) for stars with $\log g$ of 4.25. Filled symbols represent TLUSTY stars, while open symbols represent CMFGEN stars. 


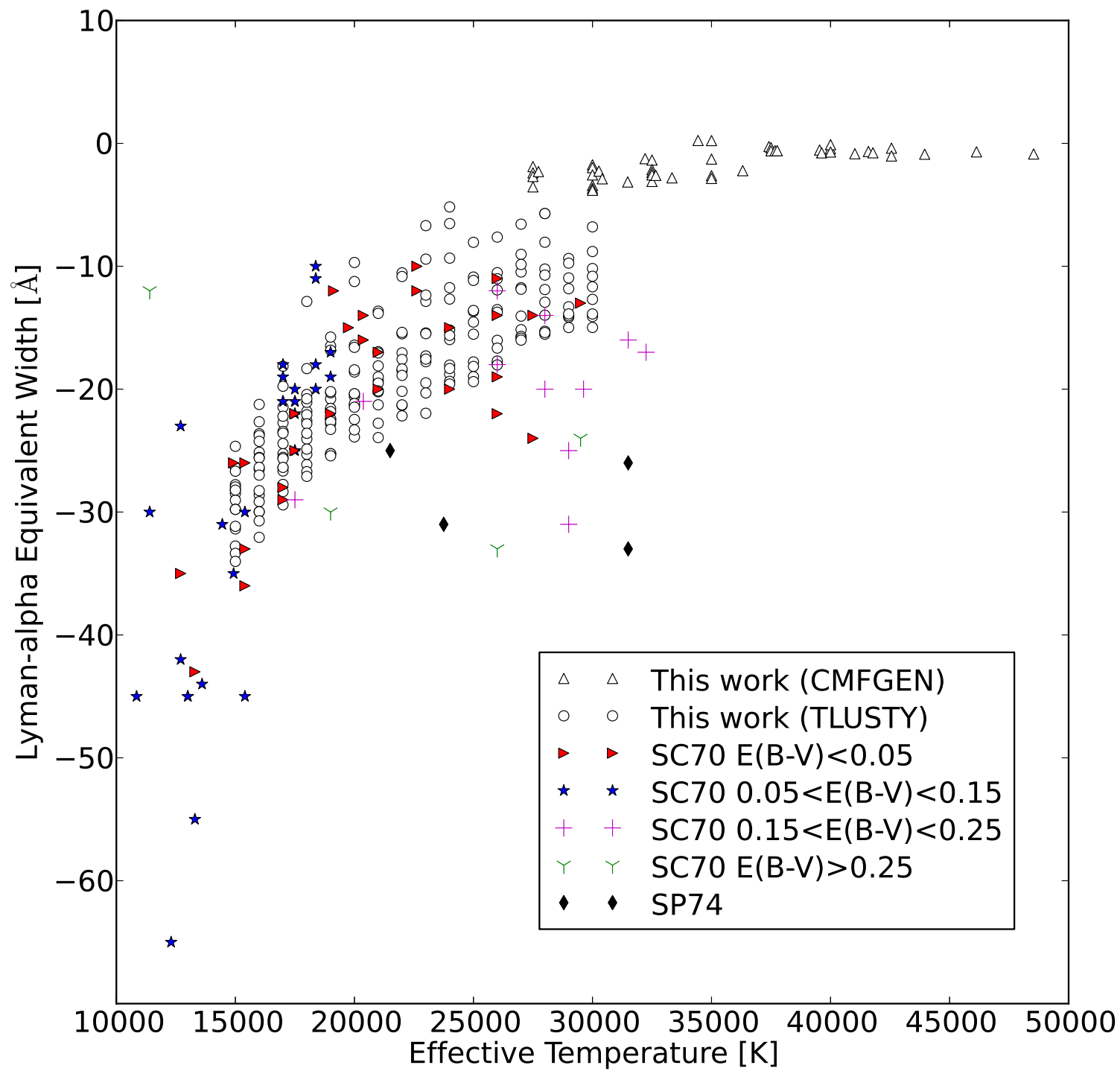

Fig. 4.- Comparison between our Ly $\alpha \mathrm{EW}$ determinations and those from observations, as a function of effective temperature. Symbols represent the following: open up triangles are EW determinations of this work, from CMFGEN models, open circles are EW determinations from TLUSTY models, red rotated triangles, magenta crosses, black diamonds, and green three-pointed stars are EW obtained from observations from Savage \& Code (1970), SC70, for different values of $E(B-V)$, and blue stars are $\mathrm{EW}$ obtained from observations from Savage \& Panek (1974), SP74. SC70 measurements are blended with the interstellar Ly $\alpha$ line and with neighboring lines, and SP74 are corrected for both ISM and blending with the Si III. 


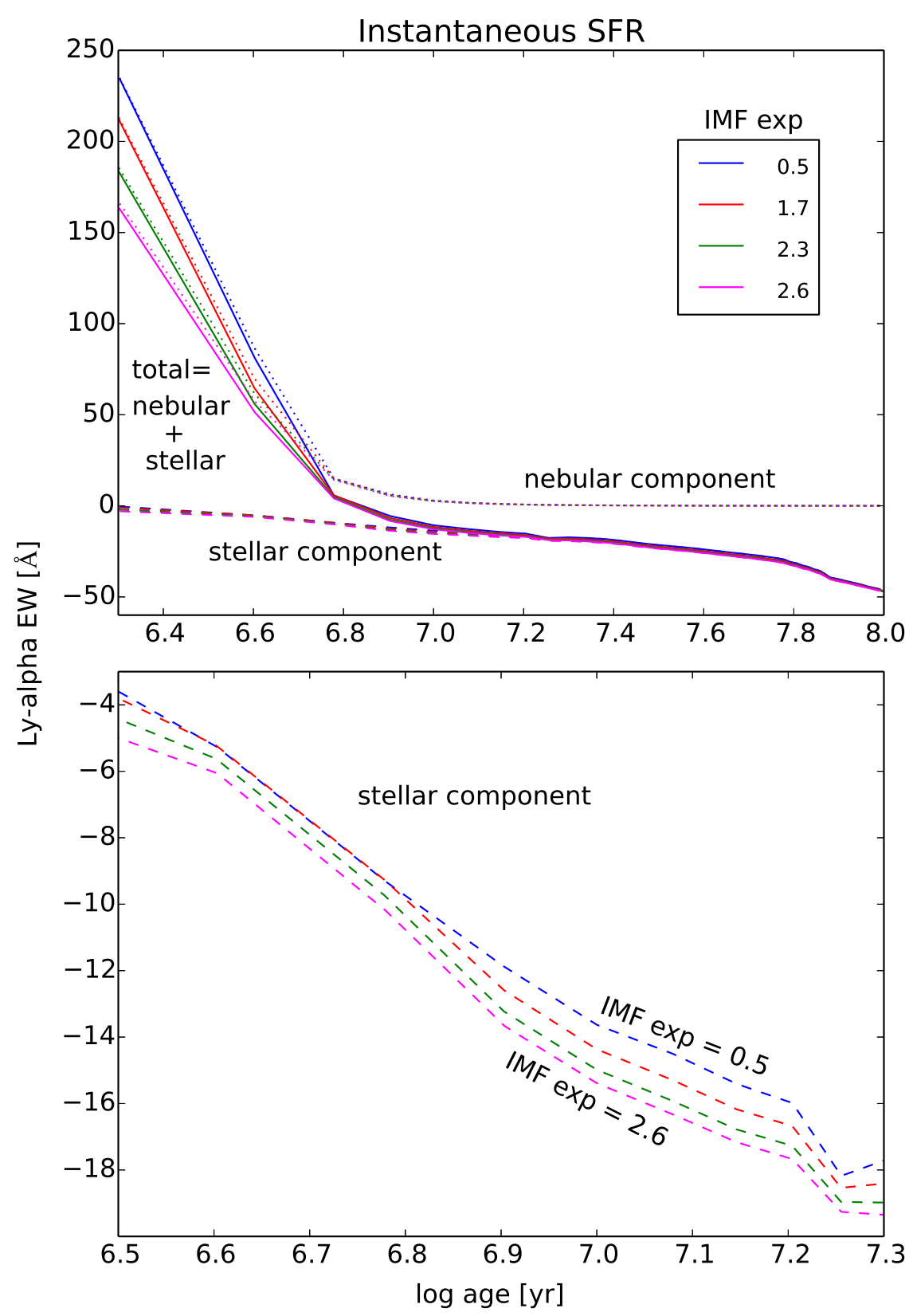

Fig. 5.- Ly $\alpha$ EW's versus age for an instantaneous burst. This figure shows a series of Starburst99 runs for different IMF exponents, labeled by color from 0.5 (blue) through 2.6(magenta). Salpeter is represented by the green curve. Dashed lines represent the stellar component of Ly $\alpha \mathrm{EW}$, dotted lines represent the nebular component, and solid lines the total component. The sign convention used for this work is positive for emission and negative for absorption. The upper panel shows the overall behavior of Ly $\alpha$ up to 100 million years. The bottom panel shows a zoom-in of the stellar component from about 3 to 20 million years. 


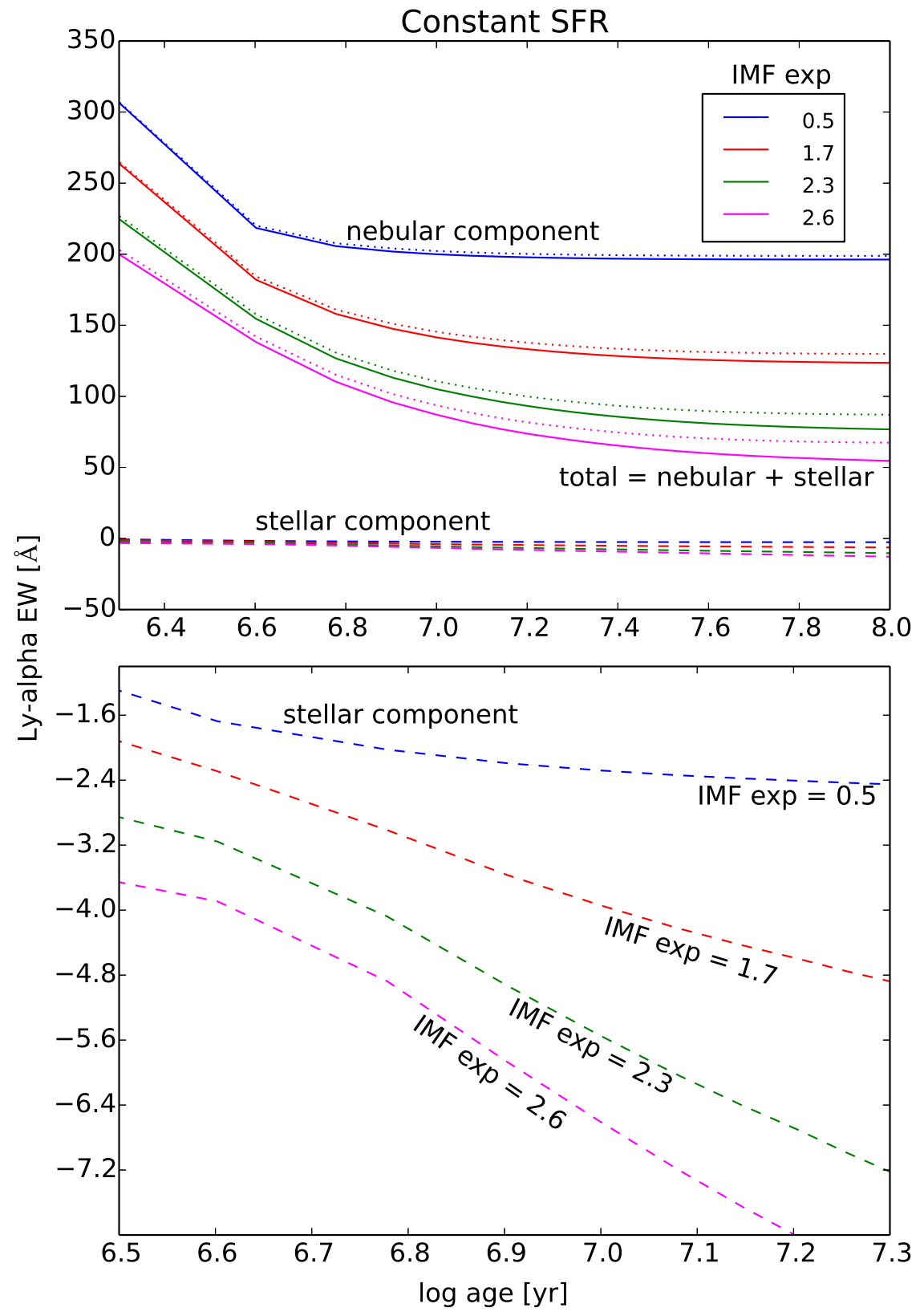

Fig. 6.- Same as Figure 5 but for constant star formation rate. 


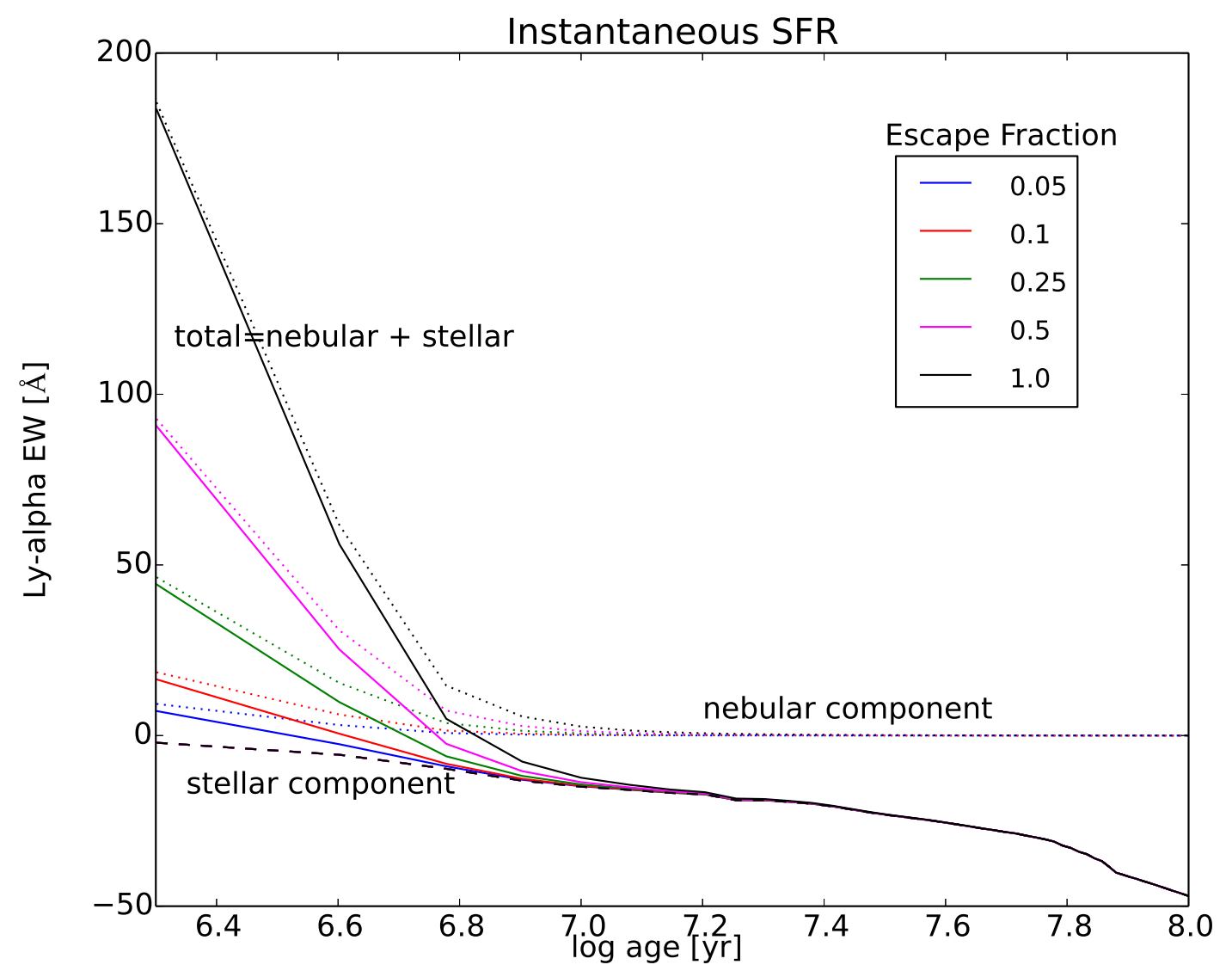

Fig. 7.- Ly $\alpha$ EW's versus age for an instantaneous burst for different Ly $\alpha$ escape fractions. This figure shows a series of Starburst99 runs for an IMF exponent of 2.3. Dashed lines represent the stellar component of Ly $\alpha \mathrm{EW}$, dotted lines represent the nebular component, and solid lines the total component. The sign convention used for this work is positive for emission and negative for absorption. 


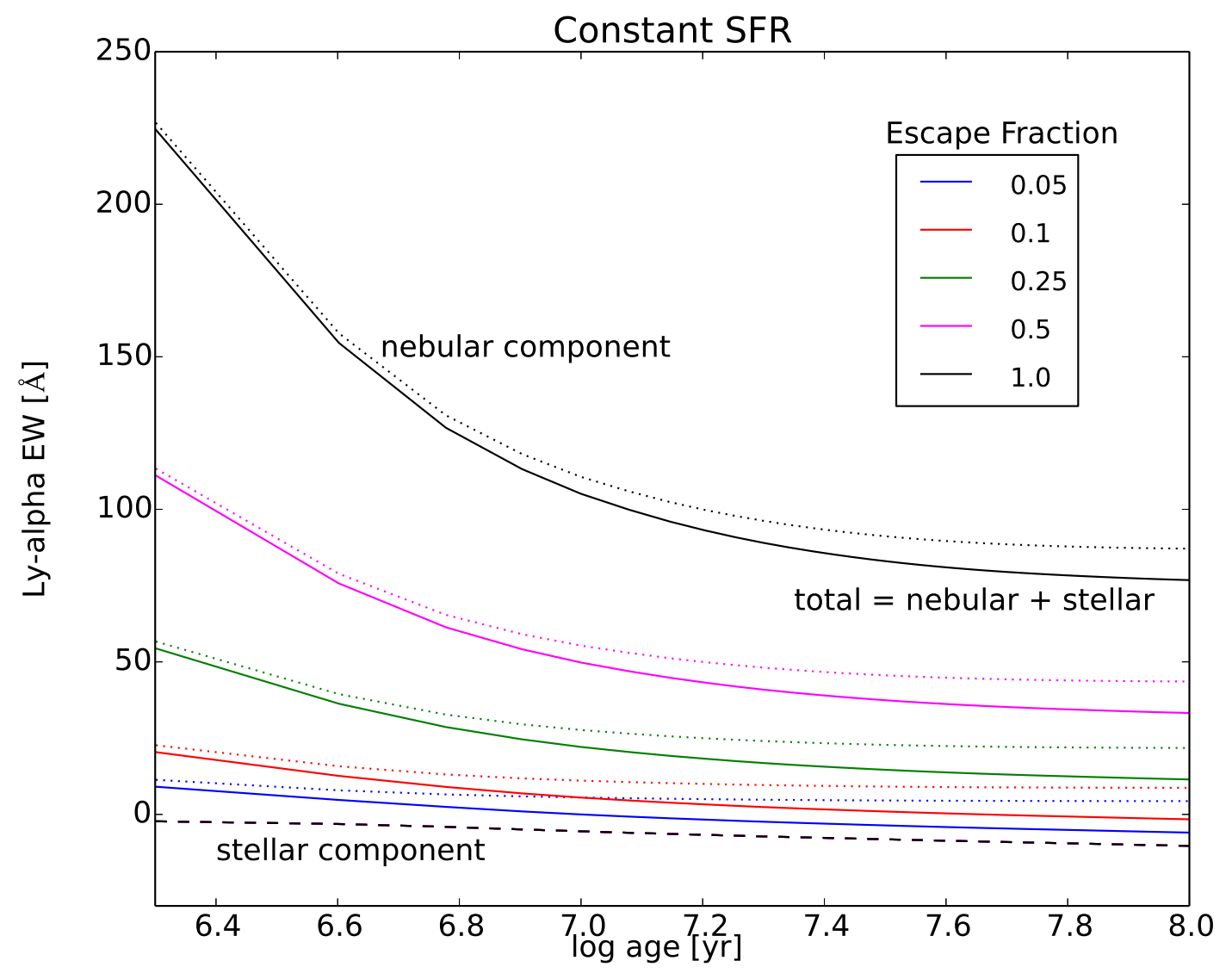

Fig. 8. - Same as Figure [7 but for constant star formation rate. 